\title{
CLINIGAL CONUNDRUM
}

The approach to clinical conundrums by an expert clinician is revealed through presentation of an actual patient's case in an approach typical of morning report. Similar to patient care, sequential pieces of information are provided to the clinician who is unfamiliar with the case. The focus is on the thought processes of both the clinical team caring for the patient and the discussant.

Jennifer Reilly Lukela, $\mathrm{MD}^{1}$

Rajesh S. Mangrulkar, MD ${ }^{1}$ Lawrence M. Tierney, Jr., MD ${ }^{2}$ John Del Valle, MD ${ }^{1}$

Sanjay Saint, MD, MPH ${ }^{1,3,4}$

${ }^{1}$ Department of Internal Medicine, University of Michigan, Ann Arbor, Michigan

${ }^{2}$ Department of Medicine, University of California at San Francisco, San Francisco, California

${ }^{3}$ Ann Arbor VA Health Services Research and Development Field Program, Ann Arbor, Michigan

${ }^{4}$ Patient Safety Enhancement Program, University of Michigan Health System, Ann Arbor, Michigan

\section{A Midlife Crisis}

1 47-year-old woman was brought to the emergency department by her family because of 1 week of abdominal pain. The pain had begun in the epigastrium but had spread across the abdomen. She described it as constant and 10 of 10 in intensity but could not identify aggravating or alleviating factors. She also complained of nausea and vomiting, beginning 4 days prior to presentation, occurring 2-5 times per day. She noted poor oral intake and mild diarrhea. She denied melena or hematochezia. She reported no recent fever, dysuria, chills, or night sweats; however, she reported upper respiratory symptoms 2 weeks prior to presentation. On the day of presentation, her family felt she was becoming increasingly lethargic.

Epigastric pain in a middle-aged woman suggests several possible diagnoses. Conditions such as acute cholecystitis begin abruptly, whereas small bowel obstruction, appendicitis, and diverticulitis start gradually. Nausea and vomiting are common concomitants of abdominal pain and are nonspecific. The absence of fever and chills is reassuring. Of greatest concern is the mental status. Initially, I think of enterohemorrhagic E. coli syndromes with associated glomerulonephritis. With a more systemic metabolic abnormality such as this, the rapid development of the disease tends to exaggerate symptoms.

The patient had a history of nephrolithiasis and underwent total abdominal hysterectomy and bilateral salpingo-oopherectomy secondary to uterine fibroids in the past. She took occasional acetaminophen, smoked two cigarettes per day, and rarely consumed alcohol. Temperature was $38.5^{\circ} \mathrm{C}$, heart rate was 160 beats/minute, respiratory rate was $28 /$ minute, and blood pressure was 92/52 mm Hg; oxygen saturation was 100\% breathing 2 L of oxygen by nasal cannula. She was a moderately obese African American woman in moderate distress, lying in bed moaning. Mucous membranes were dry. There was no lymphadenopathy or thyromegaly. Heart rate was regular without appreciable murmur, rub, or gallop. Lungs were clear. Abdomen was soft and nondistended, with diffuse tenderness to palpation; bowel sounds were present; there was no rebound or guarding. She had normal rectal tone with brown, guaiac-negative stool. There was no costovertebral angle tenderness. She was oriented to person, place, and time but lethargic; deep tendon reflexes were $3+$ bilaterally, and no focal signs were elicited. 
Renal stones certainly produce abdominal pain, and the rare patient undergoes laparotomy for this reason. The hysterectomy tells us that small bowel obstruction could be a reason for her symptoms, although abnormal mental status would not be expected without additional problems such as infection. The tachycardia seems out of proportion to her temperature. Hyperpnea and absent respiratory symptoms, along with hypotension and tachycardia, suggest a sepsis syndrome. Her physical exam confirms dehydration. Examination of the abdomen makes me speculate about whether she has a nonsurgical cause of acute abdomen. The lethargy remains unexplained. Sepsis syndrome, possibly from a perinephric abscess, is my leading diagnosis.

White blood cell count was $15.9 / \mathrm{mm}^{3}$ with $78 \%$ neutrophils, a hemoglobin of $14.3 \mathrm{~g} / \mathrm{dL}$ with a MCV of 76 and a platelet count of $320 / \mathrm{mm}^{3}$. Sodium was 159 $\mathrm{mmol} / \mathrm{L}$, chloride $128 \mathrm{mmol} / \mathrm{L}$, bicarbonate 19 $\mathrm{mmol} / \mathrm{L}$, blood urea nitrogen $120 \mathrm{mmol} / \mathrm{L}$, creatinine $3.1 \mathrm{mg} / \mathrm{dL}$, calcium $11.7 \mathrm{mg} / \mathrm{dL}$, albumin $3.3 \mathrm{~g} / \mathrm{dL}$, serum aspartate aminotransferase $65 \mathrm{U} / \mathrm{L}$, serum alanine aminotransferase $72 \mathrm{U} / \mathrm{L}$, total bilirubin 0.7 $\mathrm{mg} / \mathrm{dL}$, amylase $137 \mathrm{U} / \mathrm{L}$ (normal 30-100), and lipase 92 IU/dL (normal 4-24). Urine obtained from a Foley catheter revealed negative nitrite and leukocyte esterase, 50-75 red blood cells, and 10-25 white blood cells per high-powered field.

The elevated serum sodium is likely contributing to her abnormal mental status. It is unusual for a previously healthy and conscious woman to become this hypernatremic because persons with a normal mental status will defend their sodium balance strenuously, assuming regulatory mechanisms are intact. Generally, this level of hypernatremia indicates 2 things. One, a patient was not allowed, or did not seek access to, free water. The other is the presence of diabetes insipidus. It is unlikely she became this dehydrated from the initial gastrointestinal episode as described. The low MCV suggests she may be a thalassemia carrier, as microcytosis with iron deficiency typically does not occur until the patient is anemic, although she may be when rehydrated. Serum calcium, while elevated, also will likely return to the normal range with hydration. The metabolic abnormalities strongly suggest a problem in the central nervous system. The hematuria in the urinalysis continues to raise the possibility of nephrolithiasis as a cause of abdominal pain, though it does not fit well with the rest of the patient's clinical picture. The hematuria and pyuria both could still indicate a urinary tract infection such as pyelonephritis or perinephric abscess causing a sepsis syndrome.

An acute abdominal series and chest radiograph revealed a paucity of gas in the abdomen but no free air under the diaphragm or active cardiopulmonary disease. Abdominal ultrasound showed cholelithiasis without biliary dilation. There was no evidence of hydronephrosis, hydroureter, or perinephric abscess. A noncontrast abdominalpelvic computed tomography (CT) scan demonstrated no peripancreatic stranding or fluid collection and no nephrolithiasis or fluid collection suggestive of abscess. The admission electrocardiogram, read as sinus tachycardia with a rate of 160 , is displayed in Figure 1.

I have long believed that unexplained sinus tachycardia is one of the most ominous rhythms in clinical medicine; it is expected after vigorous exercise, among other situations, but not in the condition in which this woman finds herself. The nature of the tracing does not indicate the likelihood of a supraventricular arrhythmia, particularly atrial flutter, which should be considered given the rate. The absence of free air under the diaphragm on chest radiography is reassuring. Though the pancreatic enzymes are mildly elevated, they are usually far more striking in gallstone pancreatitis. Hypercalcemia may result in abdominal pain by several mechanisms. I remain concerned about her central nervous system.

The patient was admitted to the intensive care unit (ICU), where she received intravenous antibiotics and aggressive rehydration. The following morning, she continued to complain of abdominal pain. Her systolic blood pressure was $115 \mathrm{mmHg}$, and her heart rate ranged between 140 and 150 beats/ minute. The remainder of her physical exam was unchanged. Repeat laboratory tests revealed a white blood cell count of $14.7 / \mathrm{mm}^{3}$, a blood urea nitrogen of $66 \mathrm{mg} / \mathrm{dL}$, a creatinine of $1.3 \mathrm{mg} . \mathrm{dL}$, amylase of 67 IU/L, and lipase of $70 \mathrm{IU} / \mathrm{dL}$. A contrast-enhanced abdominal-pelvic CT scan did not reveal intra-abdominal pathology. Blood and urine cultures obtained at admission were negative for any growth.

The patient was appropriately admitted to the ICU. When caring for a critically ill patient, establishing a diagnosis is less important initially than addressing treatable conditions with dispatch. The negative CT 


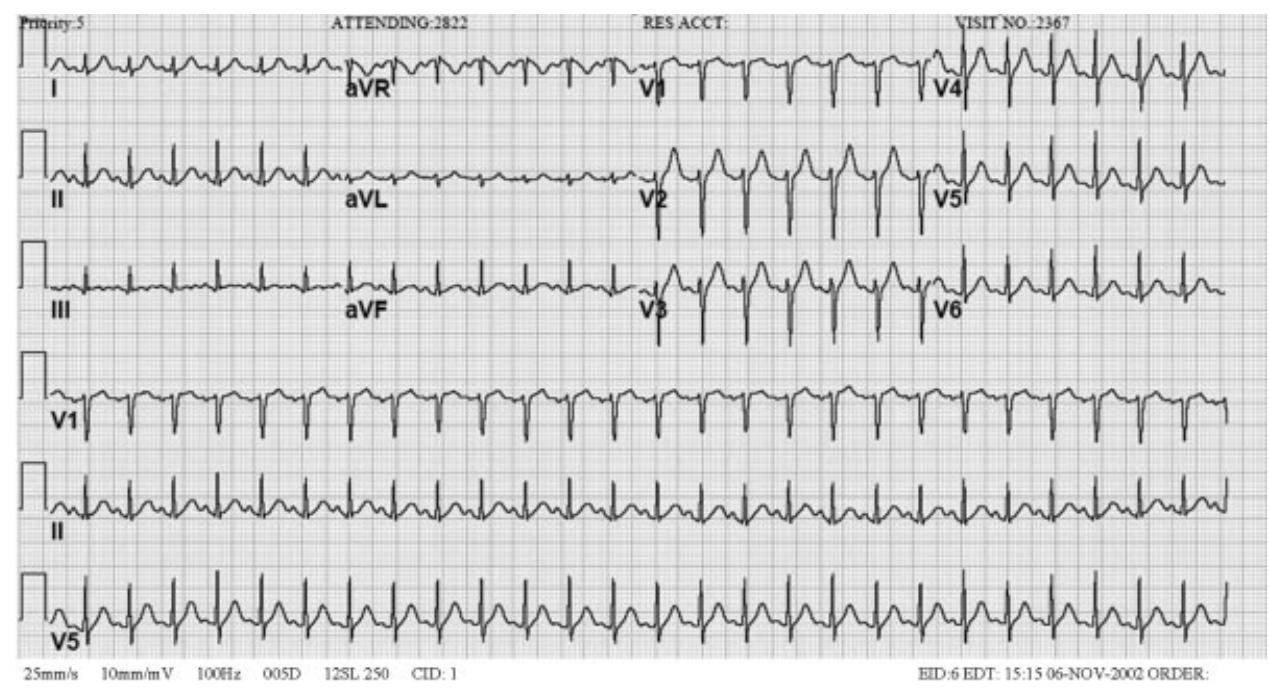

FIGURE 1. Electrocardiogram obtained at the time of admission. Interpreted as sinus tachycardia at a rate of 160 with a short PR interval.

scans rule out previously entertained diagnoses like nephrolithiasis and perinephric abscess. It is possible that the initially positive urinalysis was a result of urinary catheter placement trauma. Given the course to date, I believe this patient likely has a nonsurgical cause of abdominal pain. I am considering entities such as lead intoxication, hypercalcemia, a tear of the rectus abdominus caused by vomiting, systemic vasculitis, or a hypercoagulable state leading to intraabdominal venous thrombosis.

By hospital day 3 her sodium decreased to 149 mmol/L and her creatinine was $1.0 \mathrm{mg} / \mathrm{dL}$. Abdominal pain persisted, unchanged from admission. Her systolic blood pressure had stabilized at 120 mmHg, but the heart rate remained near 150 beats/minute. Her abdomen remained soft and nondistended on exam but diffusely tender to palpation. Her amylase and lipase continued to decrease, and her repeat electrocardiogram demonstrated tachycardia with a rate of 144.

We are gratified to see that her serum sodium has waned but not with the persistence of the tachycardia. It must be assumed that this patient has an infectious disease that we are not clever enough to diagnose at this time. I am also considering an autoimmune process, such as systemic lupus erythematosus. It is difficult to envision a neoplastic disorder causing these problems. The differential remains broad, however, because we have not ruled out metabolic or endocrine causes. It is difficult to imagine she could have Addison's disease-a com- mon cause of severe abdominal pain, tachycardia, and hypotension-given her serum sodium level. Hyperthyroidism has been known to produce mild hypercalcemia and abdominal complaints and is an intriguing possibility. The striking elevation of her serum sodium makes me consider the possibility of a problem in the posterior pituitary gland such as sarcoidosis. I cannot explain how sarcoidosis would cause her abdominal pain, unless the hypercalcemia were related. The tachycardia remains of concern, especially if she is otherwise improving. Thus, I would likely administer a small dose of adenosine to ascertain that this is not a different supraventricular tachycardia. In sinus tachycardia, the rate is usually attendant to the clinical picture and thus begs explanation given her clinical improvement.

\section{After receiving $6 \mathrm{mg}$ of intravenous adenosine, the patient's heart rate declined; atrial flutter waves were observed.}

This case nicely demonstrates a key teaching point: a fast regular heart rate of about 150, irrespective of the electrocardiogram, suggests atrial flutter. Who gets atrial flutter? Patients with chronic lung disease, myocardial ischemia (albeit rarely), alcoholinduced cardiomyopathy, and infiltrative cardiac disorders do. Additionally, we also have to consider thyroid dysfunction.

If forced to come up with a single unifying diagnosis at this point, I would have to say this patient most likely has sarcoidosis because this entity would account for modest hypercalcemia, the myo- 
cardial conduction disturbance, and hypernatremia because of diabetes insipidus; furthermore, it would fit the patient's demographic profile. However, I am also concerned about hyperthyroidism and would not proceed until thyroid function studies were obtained.

Thyroid studies revealed thyroid stimulating hormone of less than $0.01 \mathrm{mU} / \mathrm{L}$ (normal range, 0.305.50), free thyroxine (T4) of $5.81 \mathrm{ng.dL}$ (normal range, 0.73-1.79), free triiodothyronine (T3) of 15.7 pg/mL (normal range, 2.8-5.3), and total triiodothyronine (T3) of $218 \mathrm{ng} / \mathrm{dL}$ (normal range, 95-170). The patient was diagnosed with thyroid crisis and was started on propranolol, propylthiouracil, hydrocortisone, and a saturated solution of potassium iodine. Thyroid stimulating immunoglobulins were obtained and found to be markedly elevated (3.4 TSI index; normal < 1.3), suggestive of Grave's disease. Over the next several days, the patient's abdominal pain and tachycardia resolved. Her mental status returned to normal. A workup for her microcytic anemia revealed beta thalassemia trait. The patient was discharged home on hospital day 9 and has done well as an outpatient.

\section{COMMENTARY}

As Sir Zachary Cope stated in his classic text Cope's Early Diagnosis of the Acute Abdomen, "[I]t is only by thorough history taking and physical examination that one can propound a diagnosis." ${ }^{1}$ When first presented with a patient whose chief complaint is abdominal pain, physicians tend to focus on the disorders of both the hollow and solid organs of the abdomen as potential sources of the pain. The differential diagnosis traditionally includes disorders such as cholecystitis, peptic ulcer disease, pancreatitis, small bowel obstruction, bowel ischemia or perforation, splenic abscess and infarct, nephrolithiasis, diverticulitis, and appendicitis, all of which were initially considered by the clinicians involved in this case. But as our discussant pointed out, in this case the differential needed to be broadened to include less common disorders, particularly given the patient's altered mental status, numerous electrolyte abnormalities, and lethargy and the lack of explanation provided by the physical examination and sophisticated imaging studies.

Specifically, a myriad of systemic diseases and metabolic derangements can cause abdominal complaints and mimic surgical abdominal disease, including hypercalcemia, acute intermittent por- phyria, diabetic ketoacidosis, lead intoxication, familial Mediterranean fever, vasculopathies, adrenal insufficiency, and hyperthyroidism. Unfortunately, the frequency with which abdominal pain occurs in many of these less common disease processes and the pathophysiology that underlies its occurrence are not well defined. For example, abdominal pain is well described as a typical manifestation of both diabetic ketoacidosis and lead poisoning, but the pathophysiology behind its occurrence is poorly understood in both. Further, as a manifestation of thyrotoxicosis and as one of the diagnostic criteria for thyroid storm, the reported prevalence of abdominal pain in this condition is variable, ranging from "rare" to $20 \%-47 \% .^{2-4}$ Also, although other gastrointestinal manifestations of hyperthyroidism (such as nausea, vomiting, and hyperdefecation) are thought to be the result of the effect of excess thyroid hormone on gastrointestinal motility, it is unclear whether this similar mechanism is responsible for the perception of abdominal pain. ${ }^{4}$

An important clue to the underlying diagnosis in this case was the patient's marked tachycardia. Classically, a persistent heart rate of 150 should raise suspicion of atrial flutter with a 2:1 conduction block, as was eventually discovered in this case. Adenosine, in addition to other vagal maneuvers such as carotid massage or Valsalva that also block atrioventricular (AV) node conduction, has been recognized as a safe and effective means of establishing a diagnosis in tachyarrhythmias. ${ }^{5}$ In AV nodal-dependent tachycardias, such as AV node reentrant tachycardia or AV reentrant tachycardia, adenosine will often terminate the tachyarrhythmia by blocking the anterograde limb of the reentrant circuit. In AV node-independent tachyarrhythmias, such as atrial flutter or atrial fibrillation, adenosine will not terminate the rhythm. However, in the case of flutter, blocking the AV node will usually transiently unmask the underlying $\mathrm{P}$ waves, thereby facilitating the diagnosis.,

In this patient, the discovery of atrial flutter was the main clue that thyrotoxicosis may provide the unifying diagnosis. Thyroid hormone has a direct positive cardiac chronotropic effect, resulting in the increased resting heart characteristic of thyrotoxicosis. Specifically, this hormone increases sinoatrial-node firing, shortens the refractory period of conduction tissue within the heart, and decreases the electrical threshold for atrial excitation. In addition to predisposing to sinus tachycardia (the most common rhythm associated with this disor- 
der), thyrotoxicosis is also associated with atrial tachycardias such as atrial flutter and, more classically, atrial fibrillation. ${ }^{7,8}$ Though no studies have specifically evaluated the incidence of atrial flutter in thyrotoxicosis, atrial fibrillation has been found in $9 \%-22 \%$ of these patients. ${ }^{7}$

Finally, several of the patient's electrolyte derangements could explain some of her clinical findings and are clues to the underlying diagnosis. She initially presented with a mild hypercalcemia that persisted even after hydration. Potential explanations include her severe dehydration or her underlying thyrotoxicosis because hypercalcemia is present in up to $20 \%$ of patients with hyperthyroidism. ${ }^{9,10}$ However, the presence of significant hypercalcemia in the setting of thyrotoxicosis may actually make the diagnosis of thyrotoxicosis more difficult, masking the hypermetabolic signs and symptoms of the hyperthyroid state. ${ }^{11}$ Interestingly, coexistent primary hyperparathyroidism does occur in a few of these patients, but it likely was not an underlying cause in our patient given that her calcium normalized after receipt of propylthiouracil therapy. ${ }^{12}$

The patient's marked hypernatremia is more difficult to explain. She may have developed nephrogenic diabetes insipidus secondary to hypercalcemia, explained by a renal concentrating defect that can become evident once the calcium is persistently above $11 \mathrm{mg} / \mathrm{dL}^{13}$ Combined with her altered mental status, which likely limited her ability to access free water, this may be enough to explain her marked hypernatremia. Her rapid improvement with rehydration is also consistent with this explanation, mediated through the improvement of her serum free calcium.

This case highlights the importance of using all the clinical clues provided by the history, physical exam, and laboratory and imaging studies when generating an initial differential diagnosis, as well as the importance of being willing to appropriately broaden and narrow the list of possibilities as a case evolves. When this patient was initially evaluated by physicians in the emergency department, they believed her symptoms were most consistent with generalized peritonitis that was likely secondary to an infectious or inflammatory intra-abdominal process such as pancreatitis (especially in light of her mildly elevated lipase and amylase), appendicitis, or diverticulitis. When the medical team in the intensive care unit assumed care of this patient, members of the team failed to recognize several of the early clues, including the patient's markedly abnormal mental status, electrolyte derangements, and persistent tachycardia despite aggressive rehydration, which suggested the possibility of alternative, and less common, etiologies of her abdominal pain. Instead, they continued to aggressively pursue the possibility of the initial differential diagnosis, even repeating some of the previously negative studies from the emergency department. This case illustrates the importance of constantly reevaluating the available information from physical examination and laboratory and imaging studies and not falling victim to "intellectual blind spots" created by suggested diagnoses by other care providers. Fortunately for this patient, her thyroid crisis was diagnosed, albeit with some delay, before any longterm complications occurred.

Address for correspondence and reprint requests: Jennifer Reilly Lukela, MD, University of Michigan—Briarwood Medical Group, 375 Briarwood Circle, Bldg. 3, Ann Arbor, Ml 48108; E-mail: jlreilly@med.umich.edu.

Received 4 October 2005; revision received 11 February 2006; accepted 21 February 2006.

\section{REFERENCES}

1. Silen W, ed. Cope's Early Diagnosis of the Acute Abdomen. 19th ed. New York: Oxford University Press; 1995:4.

2. Harwood-Nuss AL, Martel TJ. An unusual cause of abdominal pain in young woman. Ann Emerg Med. 1991;20:574-582.

3. Harper MB. Vomiting, nausea and abdominal pain: unrecognized symptoms of thyrotoxicosis. J Fam Prac. 1989;24: 382-386.

4. Powell DW, Alpers DH, Yamada, Owyang C, Laine L, eds. Textbook of Gastroenterology. 3rd ed. Philadelphia, Pa: Lippincott Williams \& Wilkins. 1999;783, 2516.

5. Conti JB, Belardinelli L, Curtis AB. Usefulness of adenosine in diagnosis of tachyarrhythmias. Am J Cardiol. 1995;75:952-955.

6. Chauhan VS, Krahn AD, Klein GJ, Skanes AC, Yee R. Supraventricular tachycardia. Med Clin North Am. 2001;85: 193-223.

7. Woeber KA. Thyrotoxicosis and the heart. $N$ Engl J Med. 1992;327:94-8.

8. Klein I, Ojamaa K. Thyrotoxicosis and the heart. Endocrinol Metab Clin North Am. 1998;27:51-62.

9. Rude RK, Oldham SB, Singer FR, Nicoloff JT. Treatment of thyrotoxic hypercalcemia with propranolol. $N$ Engl J Med. 1976;294:431.

10. Burnam KD, Monchik JM, Earll JM, Wartofsky L. Ionized and total plasma calcium and parathyroid hormone in hyperthyroidism. Ann Intern Med. 1976;84:668.

11. Edelson GW, Kleerekoper M. Hypercalcemic crisis. Med Clin North Am. 1995;79:79-92.

12. Barsotti MM, Targovnik JH, Verso TA. Thyrotoxicosis, hypercalcemia, and secondary hyperparathyroidism. Arch Intern Med. 1979;139:661-663.

13. Rose BD, Post TW. Clinical Physiology of Acid-Base and Electrolyte Disorders. 5th ed. New York: McGraw-Hill; 2001: 754-758. 